\title{
Identification of MicroRNA-I24-3p as a Putative Epigenetic Signature of Major Depressive Disorder
}

\author{
Bhaskar Roy', Michael Dunbar', Richard C Shelton' and Yogesh Dwivedi*,I \\ 'Translational Research, UAB Mood Disorder Program, Department of Psychiatry and Behavioral Neurobiology, University of Alabama at \\ Birmingham, Birmingham, AL, USA
}

\begin{abstract}
Major depressive disorder (MDD) is predicted to be the second leading cause of global disease burden by 2030. A large number of MDD patients do not respond to the currently available medication because of its poorly understood etiology. Recently, studies of microRNAs (miRNAs), which act as a molecular switch of gene expression, have shown promise in identifying a molecular network that could provide significant clues to various psychiatric illnesses. Using an in vitro system, a rodent depression model, and a human postmortem brain, we investigated the role of a brain-enriched, neuron-specific miRNA, miR- I24-3p, whose expression is highly dysregulated in stressed rodents, and identified a set of target genes involved in stress response and neural plasticity. We also found that miR-124-3p is epigenetically regulated and its interaction with the RNA-induced silencing complex (RISC) is compromised in MDD. Using blood serum, we found similar dysregulation of miR-124-3p in antidepressant-free MDD subjects. Altogether, our study demonstrates potential contribution of miR-124-3p in the pathophysiology of MDD and suggests that this miRNA may serve as a novel target for drug development and a biomarker for MDD pathogenesis.

Neuropsychopharmacology (2017) 42, 864-875; doi:I 0.I 038/npp.2016.I75; published online 5 October 2016
\end{abstract}

\section{INTRODUCTION}

Major depressive disorder (MDD) is a debilitating disorder that leads to significant increase in morbidity and mortality (Belmaker and Agam, 2008; Bromet et al, 2011). A large number of MDD patients do not respond to the currently available medication (Warden et al, 2007; Labermaier et al, 2013). This is partially the result of a lack of understanding of the molecular circuitry underlying MDD.

It is becoming increasingly clear that disruptions across cellular networks and aberrant information processing in the circuits that regulate mood and cognition may lead to altered synaptic and structural plasticity and may be crucial in the development of MDD (Malki et al, 2015). In this context, microRNAs (miRNAs), a prominent class of small noncoding RNAs, are gaining increasing attention for their role in neural plasticity and higher brain functioning (Scott $e t a l$, 2012; Hansen et al, 2013). These miRNAs function as the master regulator of gene expression at a post-transcriptional level either by modulating the mRNA translation or transcript degradation by targeting the $3^{\prime}$-untranslated region (UTR); (Kim, 2005; Valencia-Sanchez et al, 2006).

* Correspondence: Professor Y Dwivedi, Translational Research, UAB Mood Disorder Program, Department of Psychiatry and Behavioral Neurobiology, University of Alabama at Birmingham, SC7II Sparks Center 1720 2nd Avenue South, Birmingham, AL 35294, USA, Tel: + | 205975 8459, Fax: + I 205975 8463,

E-mail: ydwivedi@uab.edu

Received 17 March 2016; revised 8 August 2016; accepted 21 August 2016; accepted article preview online 31 August 2016
Gene expression regulation by miRNAs has recently been considered to be a significant contributor to the understanding of the pathophysiology of psychiatric illnesses. This is evident from studies demonstrating altered expression of miRNAs in the brain of MDD (Smalheiser et al, 2011; Smalheiser et al, 2012; Lopez et al, 2014), schizophrenia (Smalheiser et al, 2014; Lai et al, 2016), as well as bipolar disorder (Smalheiser et al, 2014; Walker et al, 2015) patients. How these miRNAs contribute to these psychiatric illnesses, however, is not well understood. Recently, we reported that a set of miRNAs were coordinately and cohesively regulated in the prefrontal cortex (PFC) of MDD subjects, which were distinct from control group (Smalheiser et al, 2011; Smalheiser et al, 2014). In order to understand whether stress has a role in this coordinated regulation, recently we examined the effects of chronic exogenous corticosterone (CORT) administration to rats in inducing depression-like behavior and miRNA expression in PFC (Dwivedi et al, 2015). We found that CORT administration significantly increased immobility time and reduced the percentage of sucrose consumption compared with the vehicle control rats. On the other hand, there was no significant difference between chronic CORT-treated and vehicle-treated groups in total lines crossed during open-field test. When miRNA expression was examined in PFC of CORT-treated rats, we found that miRNAs had a coordinated dysregulation and a close chromosomal localization pattern, indicating their possible roles in fine tuning the outcome of the stressinduced depression phenotype. Interestingly, when examined individually, it was found that miR-124-3p was one of 
the most significantly affected miRNAs in CORT-treated rats. This brain-enriched (Sempere et al, 2004) miRNA is neuron-specific (Smirnova et al, 2005; Makeyev et al, 2007; Jovicic et al, 2013) and has been linked to synaptic plasticity (Chandrasekar and Dreyer, 2009; Fischbach and Carew, 2009) and antidepressant activity (Bahi et al, 2014).

In the present study we examined the relevance of miR-124-3p in MDD pathogenesis by investigating the following: (1) miR-124-3p-mediated regulation of putative target gene transcripts relevant to stress and synaptic plasticity; (2) endogenous binding status of miR-124-3p with target genes in vivo; (3) locus-specific origin of miR-124-3p; (4) epigenetic modulation of miR-124-3p; (5) miR-124-3p expression in PFC of individuals with MDD diagnosis $v s$ normal controls; and (6) miR-124-3p expression in serum of MDD patients.

\section{MATERIALS AND METHODS}

All studies were approved by the Animal Care Committee and Institutional Review Board of the University of Alabama at Birmingham.

\section{Animals and CORT Injections}

The study was conducted in the PFC of vehicle $(n=10)$ and CORT-treated $(50 \mathrm{mg} / \mathrm{kg}$; intraperitoneal injections for 21 days; $n=9$ ) rats. Rats were decapitated, trunk blood was collected, and brains were dissected $24 \mathrm{~h}$ after the last CORT injection between 0900 and 1100 hours as outlined earlier (Dwivedi et al, 2015). The CORT levels in serum were as follows: vehicle, $59.12 \pm 11.30$ and CORT-treated, $123.87 \pm 16.59(\mathrm{~F}=2.31, \mathrm{df}=14, p<0.001)$.

\section{Human Postmortem Brain Samples}

The study was performed in the PFC (Brodmann's area 46) obtained from depressed subjects who died from causes other than suicide $(n=15)$ and control subjects without a history of mental disorder $(n=15)$, hereafter referred to as normal controls. Brain tissues were obtained from the Maryland Brain Collection at the Maryland Psychiatric Research Center, Baltimore, MD. The brain dissection method is provided in our earlier publication (Dwivedi et al, 2006). All tissues from normal controls and depressed subjects were screened for evidence of neuropathology by experienced neuropathologists and were excluded if they exhibited features of Alzheimer's disease, infarctions, demyelinating diseases, or atrophy (or clinical history of these disorders). Toxicology and presence of antidepressants were examined by analysis of urine and blood samples from these subjects. $\mathrm{pH}$ of the brain was measured as described by Harrison et al (1995).

The psychiatric diagnosis was determined by psychological autopsy as described earlier (Dwivedi et al, 2006) using Diagnostic Evaluation After Death (DEAD) (Salzman et al, 1983) and the Structured Clinical Interview for the DSM-IV (SCID) (Spitzer et al, 1995). Normal controls were verified as free from mental illnesses using the same diagnostic procedures.

\section{Fluoxetine Treatment to Rats}

To evaluate the effects of antidepressants on miR-124-3p expression, male Sprague-Dawley rats were treated with fluoxetine $(10 \mathrm{mg} / \mathrm{kg}$, intraperitoneal for 60 days; $n=5)$ or normal saline $(n=5)$. Twenty-four hours after the last injection, brains were dissected and the expression of miR-124-3p was determined in PFC.

\section{Patient Population}

This study was performed in 17 healthy controls and 18 MDD patients. All participants were evaluated using the Mini International Neuropsychiatric Interview (Sheehan et al, 1998) or the Structured Clinical Interview for DSMIV TR (SCID) (First et al, 2002), with a second confirmatory interview by another psychiatrist. Depression severity was determined using the Montgomery-Åsberg Depression Rating Scale (Montgomery and Asberg, 1979).

Patients with any of the following characteristics were excluded: history of bipolar or any psychotic disorder, or the use of lithium or an antipsychotic within the prior 2 weeks; substance-use disorder within 3 months; uncontrolled clinically significant medical illnesses including the initiation of any new medications within 30 days; and pregnancy or lactation. Other psychiatric comorbidities were allowed as long as the MDD was considered primary. All MDD patients were psychotropic drug-free for at least 1 month before blood sampling. Healthy controls had no lifetime history of any mental disorder and were excluded for any uncontrolled clinically significant medical illnesses including the initiation of any new medications within 30 days, pregnancy, or lactation. Venous blood was collected between 0900 and 1100 hours and immediately processed for serum isolation. Serum was stored at $-80^{\circ} \mathrm{C}$ for further RNA extraction.

\section{RNA Isolation from Brain Tissues and Blood Serum}

Total RNA from rat and human PFC was isolated following TRIzol method (Invitrogen, Grandsland, NY, USA) with slight modifications as described earlier (Smalheiser et al, 2011). RNA samples were screened based on their purity $(260 / 280 \mathrm{~nm}$; cutoff $\geqslant 1.8)$ and integrity (based on agarose gel electrophoresis). Only samples showing RNA integrity number $>7$ were used.

RNA was extracted from human serum samples following the TRIzol method with few modifications. Briefly, $200 \mu \mathrm{l}$ serum was mixed with $50 \mu$ l of nuclease-free water before TRIzol addition $(750 \mu \mathrm{l})$, followed by overnight precipitation with alcohol with $40 \mu \mathrm{g}$ of glycogen as co-precipitant.

\section{First Strand cDNA Synthesis and qPCR-Based Transcript Quantification}

Relative quantification of transcripts was done following the synthesis of the first strand cDNA using $1 \mu \mathrm{g}$ of total RNA. Briefly, mRNA pool was selectively reverse-transcribed using oligo dT-based priming method. miRNA-specific first strand cDNA conversion was performed following a poly A tailing method and subsequent priming with oligo dT adopter primer (Supplementary Table S1).

Relative transcript abundance for both mRNA and miRNA was determined using EvaGreen chemistry (Applied 
Biological Material, Richmond, Canada) as described earlier (Dwivedi et al, 2015) following a delta delta Ct method (Livak and Schmittgen, 2001). The detailed primer sequence information is provided in Supplementary Table S1. Relative mRNA quantification was carried out using GAPDH as the normalizer, whereas U6 was used as normalizer for relative miRNA quantification. Both these normalizers were not significantly different in PFC of MDD subjects compared with normal controls (GAPDH: $t=0.68, \mathrm{df}=28, p=0.50$; U6: $t=1.61, \mathrm{df}=28, p=0.12)$.

\section{Luciferase Assay}

Using rat genomic DNA (gDNA), Gria4 3' UTR was PCRamplified with specific adaptor primers containing restriction digestion sites for SpeI and HindIII (Supplementary Table S1). Amplified 3' UTR was double-digested (SpeI and HindIII) and directionally cloned in PMIR luciferase reporter vector (Addgene, Cambridge, MA, USA) (Supplementary Table S1). The luciferase assay was performed by cotransfecting HEK-293 cells (293 (HEK293; ATCC CRL1573) with Gria4 3' UTR containing pMIR plasmid in three different combinations: with (i) miR-124-3p duplex, (ii) miR-124-3p hairpin inhibitor duplex, and (iii) nonspecific miR duplex (GE Healthcare Dharmacon, Lafayette, CO, USA) as negative control. Simultaneous transfection of Renilla Luciferase plasmid (Promega, Madison, WI, USA) in all three groups was carried out to analyze normalized (firefly/Renilla) luciferase activity in relative luminescence units.

\section{Transfection Assay}

Double-stranded RNA oligos, mimicking endogenous miR124-3p, antisense of miR-124-3p, and nonspecific miRNA control were transfected into SH-SY5Y neuroblastoma cell lines (SHSY5Y ATCC CRL2266). One group of control was included in the experiment as vehicle where cells were incubated with transfection reagent devoid of any RNA oligos. Cells were harvested $36 \mathrm{~h}$ after transfection for target mRNA expression analysis.

\section{RNA-Immunoprecipitation Assay}

Rat PFC (20 mg) was homogenized using polysome lysis buffer for RNA-Immunoprecipitation (RNP-IP) assay following a protocol by Keene et al (2006) with slight modifications. Briefly, prewashed protein A/G beads (Santa Cruz Biotechnology, Dallas, TX, USA) were conjugated with Ago1 monoclonal antibody (Catalogue number: 07-599; Millipore, Billerica, MA, USA) and were added with clear lysate derived from homogenized brain tissue. Following an overnight incubation, the immunoprecipitated RNA-induced silencing complex (RISC) was used to extract enriched miRNAs and their bound target mRNAs using TRIzol reagent.

First strand cDNA was reverse-transcribed from the immunoprecipitated RNA using miRNA oligo seed sequence (Supplementary Table S1). Amplified cDNA was used for miRNA-mRNA interaction analysis following qPCR assay with primer sets (Supplementary Table S1) intended for target mRNA sequence close to the miRNA-binding site at 3' UTR.

\section{Methylated DNA Immunoprecipitation Assay for pre-miR-124-3 Promoter Element}

Promoter region of pre-miR-124-3, located $1 \mathrm{~kb}$ upstream of the transcriptional unit on rat chromosome 3, was characterized for $\mathrm{CpG}$ islands. Using an in silico tool, two potential $\mathrm{CpG}$ islands were first mapped within $1 \mathrm{~kb}$ upstream region of pre-miR-124-3 transcriptional unit and then analyzed for their methylation status following methylated DNA immunoprecipitation (MeDIP) assay. Two micrograms of total gDNA isolated from rat PFC was treated with RNaseA and sheared to generate a random fragment length of $\sim 400 \mathrm{bp}$. Fragmented gDNA was first denatured and then was conjugated using Protein G magnetic beads (EMD Millipore) and $5 \mu \mathrm{g}$ monoclonal 5-methyl cytosine antibody (Zymo Research, Irvine, CA). A portion of (10\%) untreated sonicated denatured gDNA was kept separately as input control. After reverse crosslinking of immunoprecipitated complex, DNA was extracted following phenol: chloroform: isoamylalcohol $(25: 24: 1 \mathrm{~V} /$ V) method. Finally, purified DNA was subjected to qPCR using EvaGreen and primers designed to amplify $\mathrm{CpG}$ islands (Supplementary Table S1). An unmethylated control primer pair was used to determine the efficiency of immunoenrichment protocol (Supplementary Table S1). The methylation enrichment was analyzed after normalization with input control.

\section{miRNA-Specific First Strand cDNA Synthesis and qPCR in RNA Isolated from Serum}

Reverse transcription was primed on 'poly A'-attached transcripts using the oligo dT-Adapter primer (Supplementary Table S1) in $100 \mathrm{ng}$ of total RNA. Relative quantification of transcripts was carried out with prepared first strand cDNA using SybrGreen (ThermoFisher Scientific, Grand Island, NY, USA) chemistry to amplify selective miRNA with specific forward primers and a universal reverse primer (Supplementary Table S1).

\section{Statistical Analysis}

All data were analyzed using SPSS (IBM, Chicago, IL, USA). The data are reported as the mean \pm SEM. Various measures between vehicle and CORT-treated rats were analyzed by independent-sample ' $t$ ' test (confidence interval $=95 \%$ ). The relationships between target gene expression and miR-124$3 \mathrm{p}$ in vehicle and CORT-treated groups were determined by Pearson product-moment correlation analysis. The differences in the expression of target gene mRNA levels between vehicle, scramble, mimic, and anti-miR-124-3p and luciferase-based Gria4 3' UTR reporter assay between scramble, mimic, and anti-miR-124-3p were analyzed by one-way analysis of variance, followed by post hoc Tukey's multiple comparison test (Tukey's HSD). The comparison of locus-specific pre-miR-124 (miR-124-1, 124-2, 124-3) expression levels and expression of Dnmt1, 3a, and $3 \mathrm{~b}$ between vehicle and CORT-treated groups were analyzed by independent-sample ' $t$ ' test. For human postmortem brain analysis, the comparison of age, gender, brain $\mathrm{pH}$, postmortem interval (PMI), and expression of miR-124-3p as well as various target genes between control and MDD 
groups were analyzed by independent-sample ' $t$ ' test. The correlation between miR-124-3p and target genes as well as PMI, age, and brain $\mathrm{pH}$ were determined by Pearson product-moment correlation analysis. The effects of antidepressants in human postmortem tissues were evaluated by independent-sample ' $t$ ' test by comparing MDD subjects who showed presence of antidepressant toxicology at the time of death with those who did not. The two group comparisons (MDD and healthy controls) in patient population study was made using independent-sample ' $t$ ' test. Similarly, the effect of fluoxetine on miR-124-3p expression in the rat brain was analyzed using independent-sample ' $t$ ' test. An $\alpha$ level $\leqslant 0.05$ was considered statistically significant.

\section{RESULTS}

\section{Effect of Chronic CORT Administration in Rats on Expression of miR-124-3p in PFC and its Impact on Predicted Target Genes}

As reported earlier (Dwivedi et al, 2015), one of the most affected miRNAs in PFC of chronic CORT-administered rats was miR-124-3p, which showed $\sim 1.8$-fold expression upregulation. A similar pattern of upregulation ( $\sim 1.6$-fold) was noted when miR-124-3p was re-analyzed in PFC of a total of 10 vehicle controls and 9 CORT-treated rats (Figure 1a). In silico prediction analysis based on TargetScan (v.6 and 7) showed that this miRNA targets a large number of genes (Supplementary Table S2). On the basis of high context scores and strong presence of $7 / 8$ mer canonical binding motifs in the $3^{\prime}$ UTRs (Supplementary Table S3), eight highly potential genes were found as target of miR-124$3 \mathrm{p}$ whose functions were reported to be critical in brain physiology in stress and MDD pathogenesis. These include heat shock protein 90ab1 (Hsp90ab1), Akt1 substrate1 (Akt1s1), glucocorticoid receptor (Nr3c1), mineralocorticoid receptor (Nr3c2), Gria3, Gria4 (members of AMPA receptor family), and Grin2a and Grin2b (NMDA receptor family members). All of these genes were downregulated in PFC of CORT-treated rats; however, only Gria3, Gria4, Grin $2 a$, and $\mathrm{Nr} 3 \mathrm{cl}$ showed a statistically significant downregulation (Figure 1b). Moreover, the expression profile of these genes showed a pattern of inverse relationship with miR-124-3p expression in the CORT-treated group (Figure 1c-f).

\section{In silico Mapping and Characterization of $3^{\prime}$ UTR of Target Genes for miR-124-3p-Binding Sites Across Higher Vertebrates}

Figure $2 \mathrm{a}-\mathrm{p}$ shows the chromosomal localization, sequence complementarity at the $3^{\prime}$ UTR, and the evolutionary conservation of the miR-124-3p-binding site across higher vertebrates, including placental mammals of four significantly downregulated target genes (Gria3, Gria4, Grin2a, and $N r 3 c 1$ ). In silico mapping of Grin $2 a$ and $N r 3 c 1$ transcripts showed two miR-124-3p binding motifs at 3' UTRs (Figure $2 \mathrm{~g}$ and 1), whereas Gria3 and Gria4 transcripts showed only one binding motif (Figure $2 \mathrm{a}$ and $\mathrm{d}$ ). Although the Gria4 3' UTR-binding site for miR-124-3p was less evolutionarily conserved across mammalian species than Gria3 (Figure 2a and d), the transcription of Gria4 was more repressed than
Gria3 (Figure 1b). On the other hand, the level of transcriptional repression for Grin $2 a$ and $N r 3 c 1$ was much higher in the CORT-treated group than Gria3 and Gria4 (Figure 1b).

\section{Validation of miR-124-3p-Mediated Repression on Target Genes}

Mir-124-3p overexpression in SH-SY5Y neuroblastoma cell line caused $23 \%$ downregulation in the expression of endogenous GRIA4; however, the level of repression was very close to attain the statistical significance. On the other hand, a significant downregulation was found for NR3C1 (37\% repression) and $A K T 1 S 1$ (48\% repression) when miR-124-3p was ectopically overexpressed. Ectopic expression of miR-124-3p inhibitor (anti-miR-124-3p) effectively unmasked the repressive effect of endogenous miR-124-3p on GRIA4, NR3C1, and AKT1S1 mRNA levels (Figure 3a). There was a negligible repressive effect (10\%) of miR-124-3p on GRIA3 expression, whereas a $25 \%$ expression upregulation was noted with miR-124-3p antisense overexpression (Supplementary Table S8A). GRIN2A transcript remained unquantifiable in SH-SY5Y cell line (Supplementary Table S7B), which is consistent with the Human Protein Atlas Database (Uhlen et al, 2015).

To establish the direct effect of miR-124-3p on Gria4 expression, a luciferase reporter assay was performed using HEK-293 cell line. The wild-type 3' UTR of Gria4 mRNA with a putative binding side for miR-124-3p was cloned upstream to luciferase reporter gene in the pMIR reporter vector backbone (Figure $3 \mathrm{~b}$ ). Simultaneous transfection of the $3^{\prime}$ UTR reporter clone along with mimic miR-124-3p oligo duplex resulted in significant downregulation of reporter gene expression compared with nonspecific oligo duplex (scramble)-transfected control (Figure 3c). The opposite effect on luciferase reporter expression was noted as a function of miR-124-3p inhibitor overexpression where luciferase activity was increased significantly as compared with the nonspecific oligo-transfected control (Figure 3c).

\section{RNP-IP to Identify Endogenous Binding of miR-124-3p with Target Genes in CORT-Treated Rat PFC}

The immunoprecipitated RISC was used to isolate RNA and was subjected to $\mathrm{qPCR}$ amplification using a miR-124-3p seed sequence and a set of $3^{\prime}$ UTR-specific primer pairs recognizing two miR-124-3p-binding sites (7mer-A1 and 7mer-m8; proximal and distal from stop codons) for $\mathrm{Nr} 3 \mathrm{cl}$, one for Gria4, and one for Akt1s1 transcript, one for Gria3 and one for Grin2a (Supplementary Table S3). To identify that the miR-124-3p-Nr3c1 and miR-124-3p-Gria4 mRNA complexes were indeed precipitated, a nonspecific primer pair designed from coding region for the Gapdh gene was used (Supplementary Table S8C). A threefold binding enrichment for miR-124-3p on Gria4 3' UTR was found in the CORT-treated group (Figure $3 \mathrm{~d}$ ). The binding enrichment for miR-124-3p on Nr3c1 transcript in the CORTtreated group was ninefold, which was confined to the $7 \mathrm{mer}$ m8 site. No change was noted on the 7 mer-A1 site (Figure 3e). Likewise, no significant binding enrichment was captured for the $3^{\prime}$ UTR of Akt1s1 transcript with miR-124-3p (Figure 3f). The binding enrichments for 
a

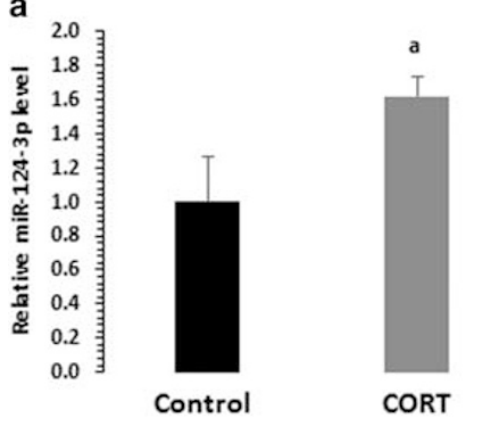

c
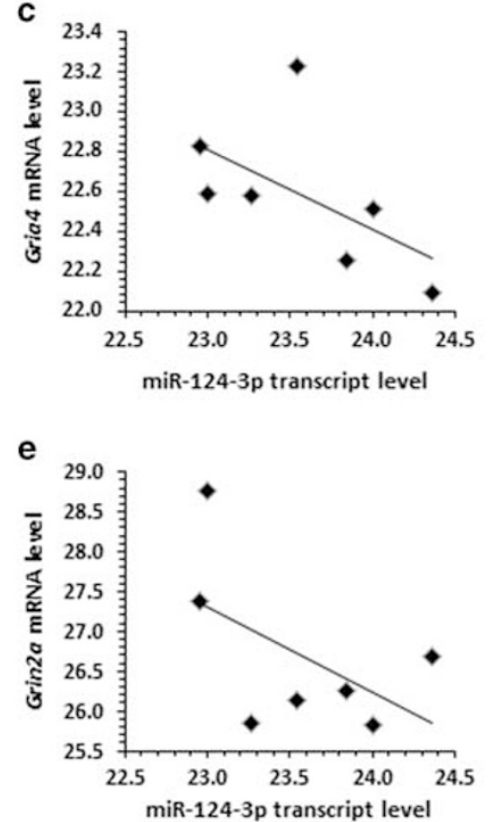

b

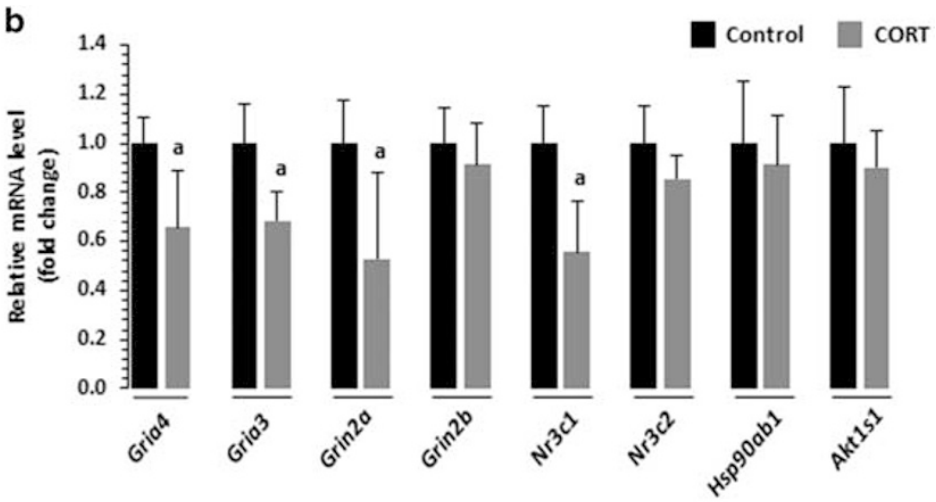

d

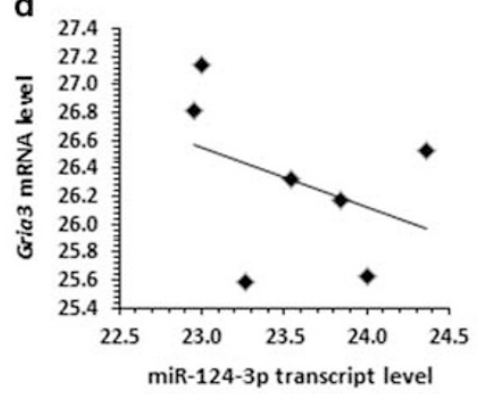

f

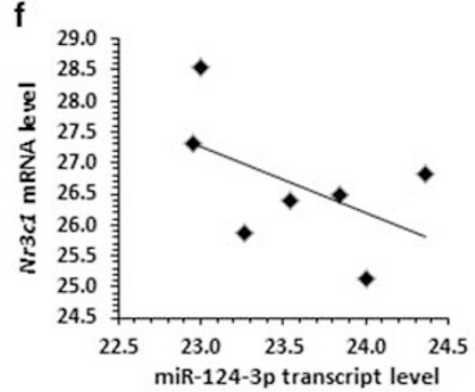

Figure I In vivo expression profiling of miR- I24-3p and its target genes in corticosterone (CORT)-treated rat prefrontal cortex (PFC). (a) Relative transcript abundance of mature miR-124-3p in CORT-treated rat PFC, data normalized to U6 expression, and values are represented as \pm SEM. Statistical significance determined by independent-sample ' $t$ ' test ( $n=10$ in control, 9 in treatment; $p=0.032$ ). (b) Relative fold change of Gria4, Gria3, Grin2a, Grin2b, Nr3cl, Nr3c2, Hsp90ab I, and Akt/s I transcripts in CORT-treated rat PFC over vehicle control group. Data are normalized with Gapdh expression, values are represented as \pm SEM, and level of significance was determined by independent-sample ' $t$ ' test $(n=5$ in each group for Gria4 $p=0.045, \mathrm{Nr} 3 \mathrm{cl} p=0.0 \mathrm{I} I, \mathrm{Hsp} 90 \mathrm{ab} / \mathrm{l}=0.70$, and Akt/s $\mid p=0.59 ; n=6$ in control and $n=5$ in CORT group for Gria3 $p=0.02$, Grin2a $p=0.03$, Grin2b $p=0.52$, and Nr3c2 $p=0.27$ ). ( $c-f$ ) Correlation between miR-124-3p and Gria4, Gria3, Grin2a, and Nr3cl genes in rat PFC were analyzed by Pearson product-moment analysis, which demonstrates a negative trend under chronic CORT treatment determined by qPCR assay (data represented as Ct values corresponding to abundance of transcripts, Gria4, $r^{2}=0.33, p=0.17, n=6$; Gria3, $r^{2}=0.15, p=0.38, n=6$; Grin2a, $r^{2}=0.29, p=0.21, n=6$; and Nr3cl, $r^{2}=0.27, p=0.23, n=6$ ). 'a' denotes significant difference between vehicle and CORT-treated groups.

miR-124-3p on Gria3 and Grin2a transcripts were increased in the CORT-treated group but they failed to reach statistical significance (Figure $3 \mathrm{~g}$ and $\mathrm{h}$ ).

\section{Locus-Specific Origin of Mature miR-124-3p}

We mapped the candidate promoter element(s) responsible for observed miR-124-3p transcriptional upregulation. For this, we analyzed the relative expression of three miR-124 precursors originating from the following three different chromosomal loci: pre-miR-124-1 (chromosome 15), premiR-124-2 (chromosome 2), and pre-miR-124-3 (chromosome 3). As shown in Figure 4a, the relative transcript abundance of precursor miR-124-3 was significantly downregulated $(\sim 50 \%)$ in the CORT-treated group without any change in pre-miR-124-1 and pre-miR-124-2.

\section{MiR-124-3 Gene Promoter Analysis to Identify Putative CpG Islands and their Possible Involvement in DNA Methylation, Mediated by Dnmts in CORT-Treated Rat PFC}

Using 'MethPrimer' in silico CpG site prediction algorithm (Li and Dahiya, 2002), we identified the presence of two CpG islands in close vicinity on miR-124 gene promoter located on chromosome 3 (Figure 4b). The first CpG island (Island1) comprises 751 bases, whereas the second one (Island 2) encompassed 108 bases. The overall GC contents for these two islands were more than $50 \%$ (Figure $4 \mathrm{~b}$ ). The possible 
a

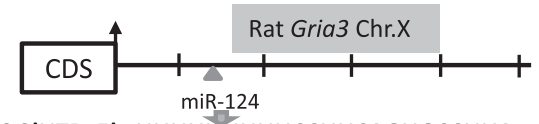

b Gria3 3'UTR 5'...UUUUUUUUUUCCUUCAGUGCCUUA

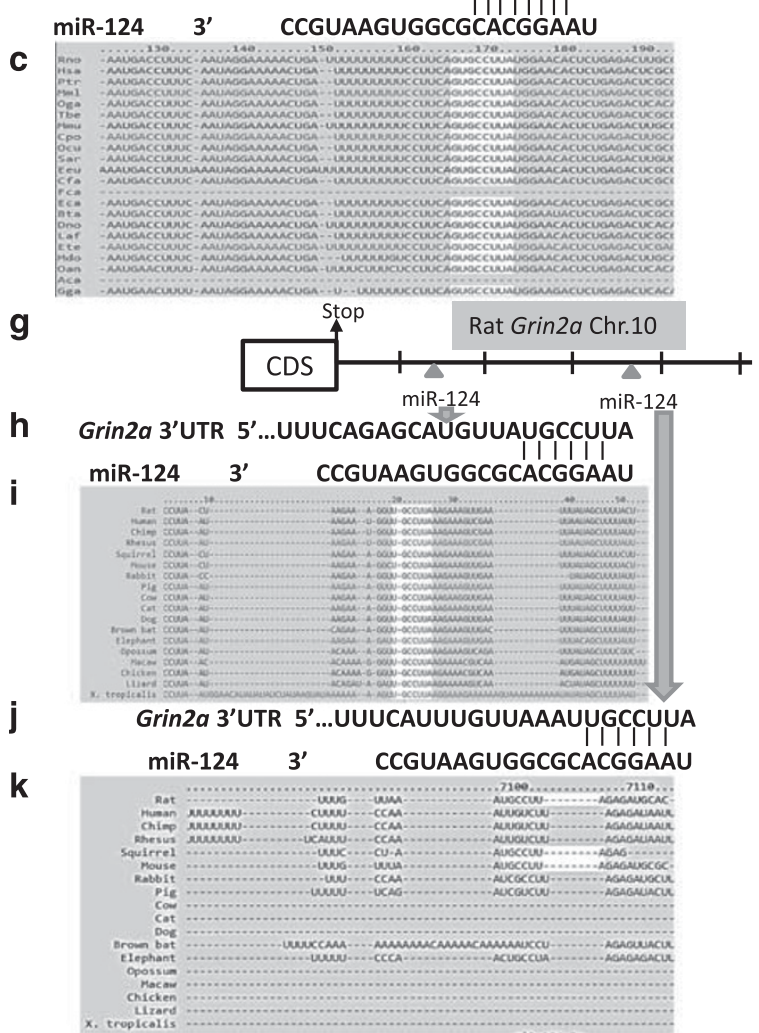

d

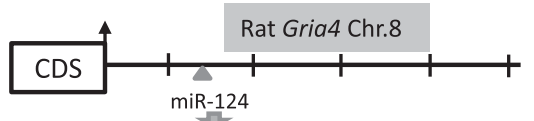

e Gria4 3'UTR 5'...CAAAÁÁAUUAAUUGAgUGCYYYUA

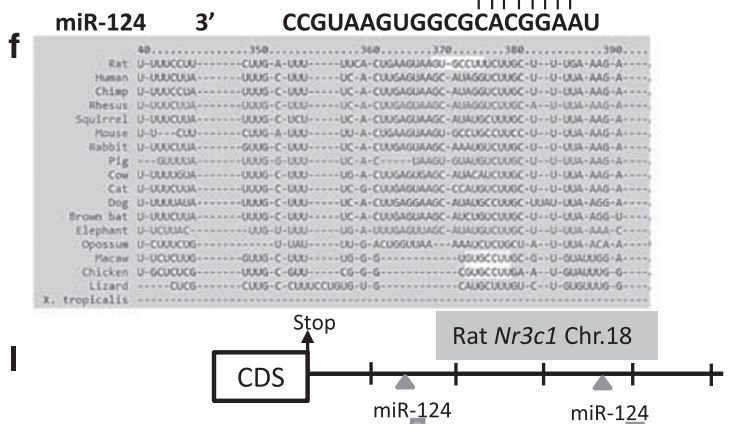

m Nr3C1 3'UTR 5'...CUUACUAAGAAAGGUUGCCUUAA miR-124 3' CCGUAAGUGGCGCACGGAAU

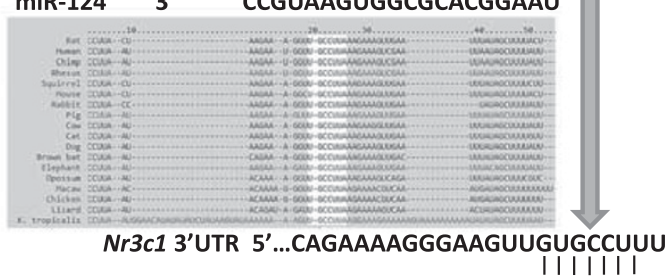

p

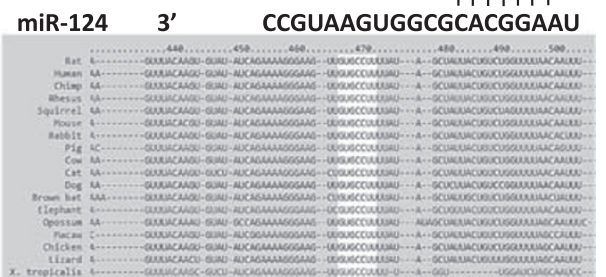

Figure 2 Map of miR-124-3p-binding sites on 3' untranslated region (UTR) of target gene(s) with sequence conservation. (a-c) Representation of the single miR-124-3p-binding site on 3' UTR of Gria3 mRNA transcribed from rat chromosome X (Chr. X) with an evolutionary conservation pattern across vertebrate species. (d) In silico mapping of the single unique miR-124-3p-binding site on Gria4 3' UTR. (e) Mapping of 7mer-m8 canonical seed match for miR-I24-3p binding on 3' UTR of rat Gria4 transcript. (f) Species-wide homology analysis of the miR-I24-3p-binding site on Gria4 3' UTR across different vertebrate representatives. (g) Mapping of Grin2a transcript from rat chromosome 10 (Chr. I0) demonstrated the presence of two miR-I24-3p-binding sites on 3' UTR. (h) The closest binding site from termination codon was a 7mer-AI canonical motif showing sequence conservation pattern (i) for miR-I24-3p binding across different vertebrate species. (j) Representation of mapping of a second 7mer-AI canonical binding motif for miR-I24-3p seed sequence on Grin2a transcript identified farthest from stop codon. (k) Representative of sequence homology pattern across distant vertebrate taxa for the second 7mer-AI site on Grin2a 3' UTR for miR-124-3p. (I) 3' UTR scanning represents two miR-I24-3p-binding sites on rat Nr3cl transcript expressed from chromosome I8 (Chr. I8). (m) Exhibition of a proximal miR-I24-binding site from CDS on Nr3cl 3' UTR with a 7mer-AI canonical sequence motif. (n) Demonstration of seed match sequence conservation for miR-124-3p identified across broad spectrum of vertebrate species on Nr3cl 3' UTR. (0) Demonstration of the mapping of a second canonical '7mer-m8' motif for miR- I24-3p binding on 3' UTR of Nr3cl transcript. (p) Representative of multiple sequence alignment for Nr3cl 3' UTR across vertebrate species for miR-124-3p binding on 7mer-m8 canonical motif.

functional contribution of these two potential CpG islands on miR-124-3 gene promoter was determined using MeDIP assay. As shown in Figure 4c, the miR-124-3 promoter was hypomethylated ( 0.7-fold less enrichment) in the CORTtreated group compared with the control group (Figure 4c).

To examine whether the observed promoter hypomethylation of miR-124-3p was associated with altered expression of DNA methylation enzymes, transcript levels of DNA methyltransferase (Dnmt) 1, Dnmt3a, and Dnmt3b were determined. We found that expression of Dnmt3a was significantly repressed in PFC of CORT-treated rats without any change in Dnmt1 and Dnmt3b (Figure 4d).

\section{miR-124-3p and Target Genes in PFC of MDD Subjects}

The demographic characteristics of control $(n=15)$ and MDD $(n=15)$ subjects are given in Supplementary Table S4.
In PFC of these subjects, expression of miR-124-3p and its target genes were examined. As shown in Figure 5a, there was a significant increase in the expression of miR-124-3p in the MDD group. On the other hand, expressions of GRIA3, GRIA4, and NR3C1 were significantly lower in the MDD group, except that of GRIN2A variants (GRIN2A I and GRIN2A II), which was lower but did not reached the significance level (Figure 5b).

There was no significant effect of gender (Supplementary Figure S1A), race (Supplementary Figure S1B), PMI and brain $\mathrm{pH}$ (Supplementary Table S5) on expression of miR-124-3p or target genes. On the other hand, age was positively correlated with miR-124-3p expression, whereas it had no effects on any of the target genes studied (Supplementary Table S5). To examine whether antidepressants had an impact on the expression of miR-124-3p or target genes, we divided MDD subjects into those who 
b

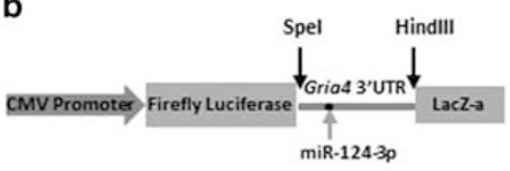

a

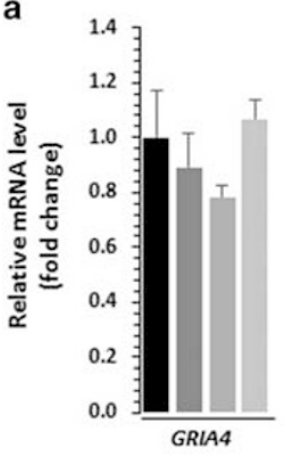

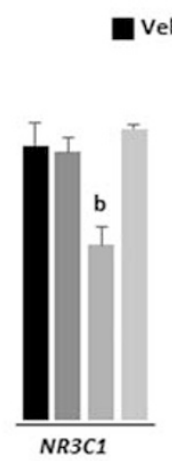

Vehicle

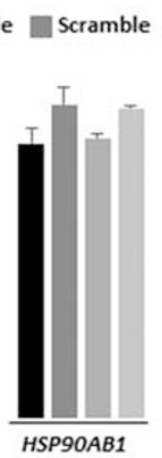

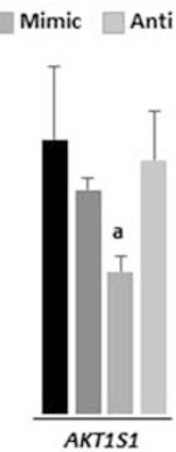

C
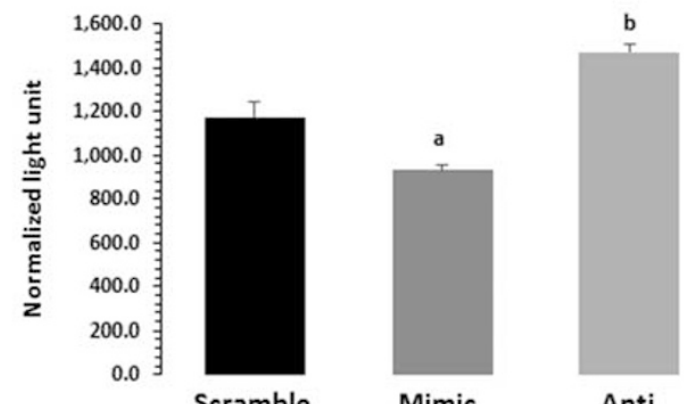

d
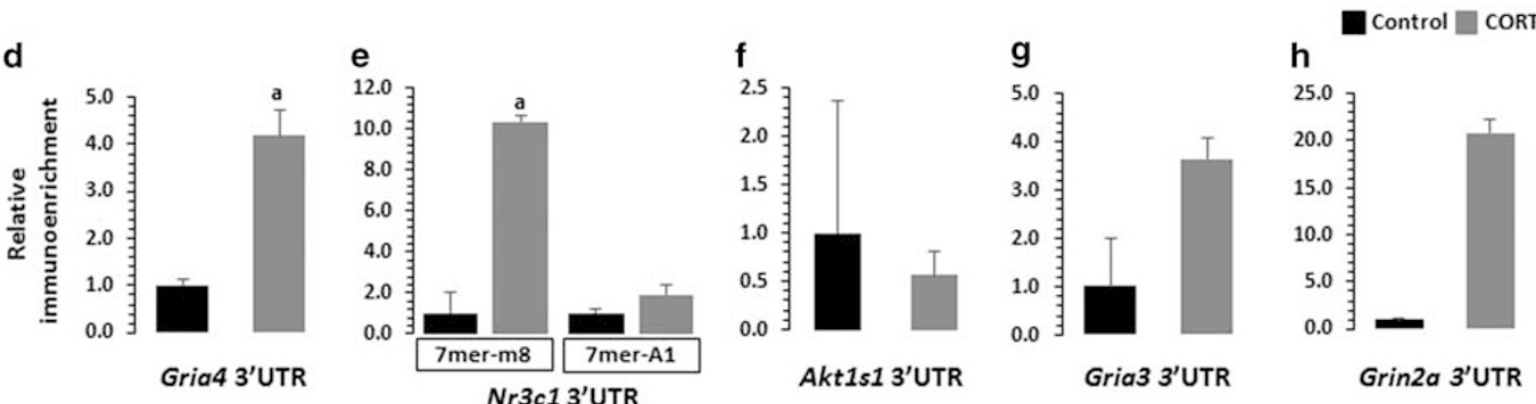

Figure 3 Functional validation of miR-I24-3p targets. (a) Bar diagram represents the relative quantification of GRIA4, NR3CI, HSP9OABI, and AKTISI transcripts in SH-SY5Y neuroblastoma cell line transiently transfected with non-target-specific scramble control $(n=3)$, mimic miR-I24-3p overexpression oligo $(n=3)$, anti-miR- I 24-3p overexpression oligo $(n=3)$, and vehicle control $(n=3)$. The expression status of respective genes was quantified in treatment groups relative to vehicle control using one-way analysis of variance, followed by post hoc Tukey's multiple comparison test. GAPDH was used as normalizer for target gene expression. Values are represented as \pm SEM ' $a$ ' and 'b' denote significant differences when compared with vehicle. Overall group differences in the four groups (vehicle, scramble, mimic, and anti) are as follows: GRIA4: $\mathrm{df}=3.8 ; f=2.95, p=0.09 ; \mathrm{NR} 3 \mathrm{Cl}: \mathrm{df}=3.8 ; f=32.15, p<0.00 \mathrm{l} ; \mathrm{HSP} 90 \mathrm{ABI}: \mathrm{df}=3.8$; $f=4.92, p=0.03 ;$ AKTISI: $d f=3.8 ; f=6.61, p=0.015 .{ }^{a} p<0.029 ;{ }^{b} p<0.001$. (b) Schematic representation of Gria4 3' untranslated region (UTR) cloning strategy with restriction enzyme digestion (Spel and Hindlll restriction enzyme digestion sites) in pMIR-Report vector downstream of firefly luciferase reporter gene. (c) Relative luciferase activity (normalized with Renilla luciferase activity) driven by Gria4 3' UTR via miR-I 24-3p interaction was determined in HEK-293 lysate co-transfected with reporter clone and individually mimic miR-I24-3p oligo $(n=3)$ or antisense (anti) miR-124-3p oligo $(n=3)$. Representative data were compared with nonspecific scramble control $(n=3)$ and expressed as \pm SEM. Overall group differences in the three groups (scramble, mimic, and anti) are as follows: $\mathrm{df}=2.6 ; \mathrm{f}=33.22, \mathrm{p}=0.00 \mathrm{I}{ }^{\mathrm{a}} \mathrm{p}=0.023 ;{ }^{\mathrm{b}} \mathrm{p}=0.0 \mathrm{II}$. ( $\left.\mathrm{d}-\mathrm{h}\right)$ Involvement of endogenous miR-124-3p binding on single $7 \mathrm{mer}-\mathrm{m} 8$ site of Gria4, two different sites of $\mathrm{Nr3} \mathrm{cl}$, one 8mer site of Akt Is I, one 8mer site of Gria3, and the proximal 7mer-AI site of Grin2a on their 3' UTR determined by RNAinduced silencing complex (RISC)-mediated immunoenrichment assay. Relative 3' UTR enrichment normalized to I0\% input in the immunoprecipitated ribonucleoprotein complex was analyzed with qPCR. Data are represented as \pm SEM. Data were analyzed by independent-sample ' $t$ ' test. 'a' denotes significant difference between vehicle and corticosterone (CORT)-treated groups. Gria $4 p=0.022, \mathrm{Nr} 3 \mathrm{cl} 7 \mathrm{mer}-\mathrm{m} 8 \mathrm{p}=0.034, \mathrm{Nr} 3 \mathrm{cl} 7 \mathrm{mer}-\mathrm{Al} p=0.133$, Akt/s I $p=0.58$, Gria3 $p=0.16$ and Grin2a $p=0.39$.

showed positive antidepressant toxicology and those who did not. The comparison analyses found no significant differences between these two groups on the expression levels of any of the genes measured (Supplementary Figure S1C). We also examined expression of miR-124-3p in PFC of fluoxetine-treated rats and found that its expression was significantly downregulated ( $35 \%$; Figure $5 c)$.

\section{Expression of miR-124-3p in MDD Patients}

The expression of miR-124-3p was studied in the serum of antidepressant-free MDD subjects and matched healthy controls (Supplementary Table S6). Comparative analysis showed that the expression of miR-124-3p was $\sim 3.5$-fold higher in MDD patients, which was statistically significant
(Figure 5d). There were no significant effects of age (Supplementary Figure S2A), gender (Supplementary Figure S2B), or race (Supplementary Figure S2C) on expression of miR-124-3p.

\section{DISCUSSION}

In a recent study, we demonstrated that depressive behavior induced by chronic CORT administration caused significant changes in miRNA expression in PFC (Dwivedi et al, 2015), a brain area critically involved in the regulation of glucocorticoid feedback inhibition and maintenance of HPA axis equilibrium in response to stress (Jankord and Herman, 2008; Hall et al, 2015). In the present study, we 
a

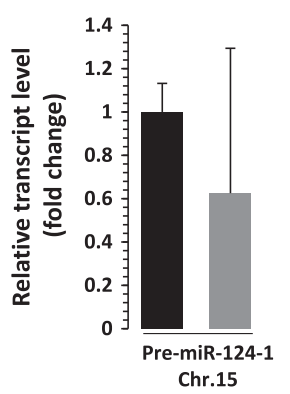

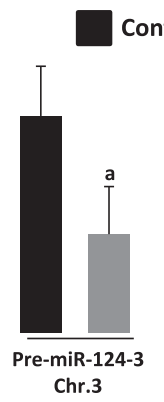

Control

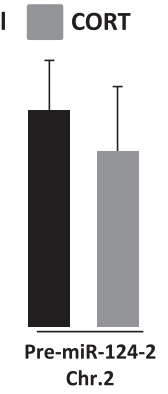

C

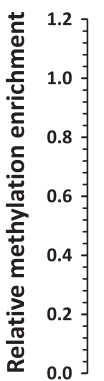

miR-124-3 promoter on rat Chr.3

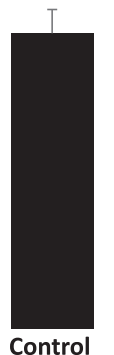

b

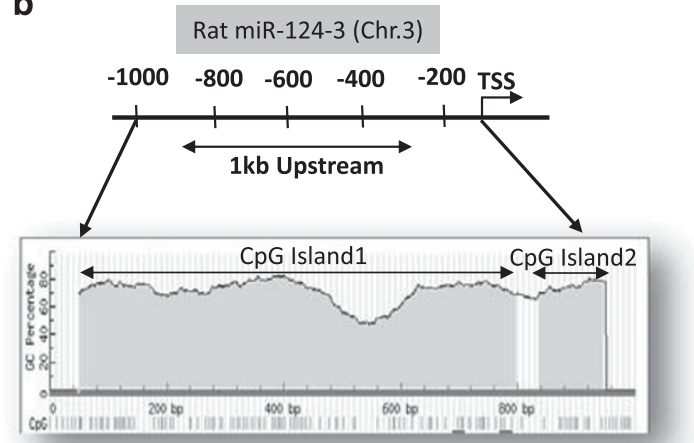

Figure 4 Effect of chronic corticosterone (CORT) treatment on transcriptional regulation of the miR-I24 gene. (a) U6-normalized relative expression values for precursor miR-124-I, precursor-miR-124-3, and precursor miR-I24-2 transcribed from three different chromosomal loci of rat prefrontal cortex (PFC) administered with chronic corticosterone. All data demonstrate the mean \pm SEM from five rats in each group and independent-sample $t$-test was used to analyze the statistical significance. $p$-value for precursor-miR- $124-3$ was 0.007 , whereas no significant differences were found for pre-miR- $|24-|$ ( $p=0.34$ ) and pre-miR- I 24-2 ( $p=0.44)$. (b) Schematic diagram represents the in silico-analyzed data for miR- 124 proximal promoter (I kb upstream from transcription start site) on rat chromosome 3 (Chr. 3). MethPrimer-based CpG site prediction algorithm identified two CpG islands on miR-I 24 proximal promoter with presence of more than 50\% GC content. (c) Graph shows the relative methylation enrichment of the miR- 124 promoter on Chr. 3 in CORT-treated rat PFC as compared with vehicle control. Represented data were normalized with 10\% input and expressed as fold change over control group. Results are mean \pm SEM obtained from three independent experiments $(n=3$ rat per group, $p=0.000$ I). (d) Gapdh normalized relative expression level of Dnmtl, Dnmt3a, and Dnmt3b transcripts were analyzed in CORT-treated rat PFC and compared with the vehicle control group. All data demonstrate the mean \pm SEM from five rats in each group and independent-sample ' $t$ ' test was used to analyze the statistical significance. 'a' denotes significant differences between vehicle and CORT-treated groups. Dnmt3a showed significant difference $(p=0.05)$, whereas no significant differences were found for Dnmtl $(p=0.13)$ and Dnmt3b $(p=0.21)$.

examined one of the most significantly upregulated miRNAs by CORT, i.e., miR-124-3p, which is not only developmentally regulated but also has a significant role in maintaining neuronal cell identity (Maiorano and Mallamaci, 2010) and synaptic plasticity (Rajasethupathy et al, 2009).

We first determined the contributory pre-miRNAs, which may be responsible for observed miR-124-3p transcriptional upregulation under CORT treatment. Our qPCR analysis showed significant involvement of pre-miR-124-3 located on chromosome 3 compared with pre-miR-124-1 or premiR-124-2 in forming the mature miR-124-3p in cytosol. We next examined the factors responsible for increased expression of miR-124-3p in CORT-treated rat PFC. In silico characterization of miR-124-3 promoter on rat chromosome 3 mapped the presence of two potential $\mathrm{CpG}$ islands within the proximal area (1 kb upstream) from transcription start site. Using MeDIP analysis, we observed that two CpG islands present in close vicinity of the miR-124-3 gene transcriptional start site were hypomethylated in PFC of CORT-treated rats, suggesting epigenetic regulation of miR-124-3p expression via altered promoter methylation. Interestingly, we also found that expression of Dnmt3a was significantly repressed without any change in Dnmt1 or 3b, suggesting that maintenance but not de novo DNA methylation is compromised, which could partially be responsible for promoter hypomethylation of miR-124-3 in PFC of CORTtreated rats (Oka et al, 2006; LaPlant et al, 2010; Barbier et al, 2015). Moreover, we found that the promoter region of miR-124-3p contained $\mathrm{Nr} 3 \mathrm{cl}$ (glucocorticoid receptor)binding sites (Dwivedi et al, 2015), which regulate miR-124-3 in a positive manner. However, our ChIP analysis showed no significant differences in the interaction of glucocorticoid receptor with the mapped $\mathrm{Nr} 3 \mathrm{cl}$-binding sites between CORT-treated and control groups (Supplementary Table S8D), suggesting the possibility that miR-124-3p may not be directly regulated by $\mathrm{Nr} 3 \mathrm{cl}$ under chronic CORT treatment.

Our initial screening of possible target genes based on high prediction scores and presence of strong $7 / 8$ mer sites at the $3^{\prime}$ UTR of the coded transcripts led us to choose eight genes that are involved in various physiological functions in brain including synaptic plasticity (Meador-Woodruff et al, 2001; Sprengel, 2006; Sagata et al, 2010; Wei et al, 2012; Duric et al, 2013; Dwyer and Duman, 2013; Medina et al, 2013; Ota et al, 2014; Hall et al, 2015). These included Gria3 (glutamate receptor, ionotropic, Ampa3), Gria4 (glutamate receptor, 
a

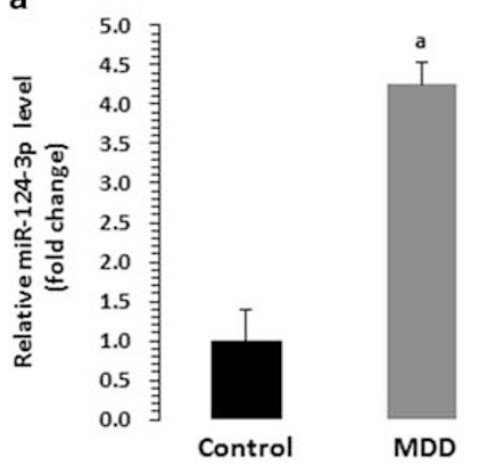

b

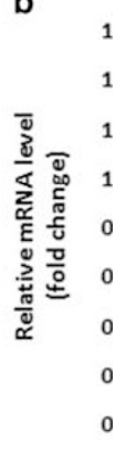

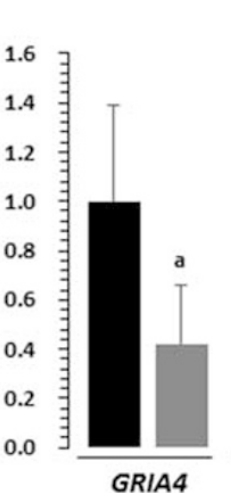
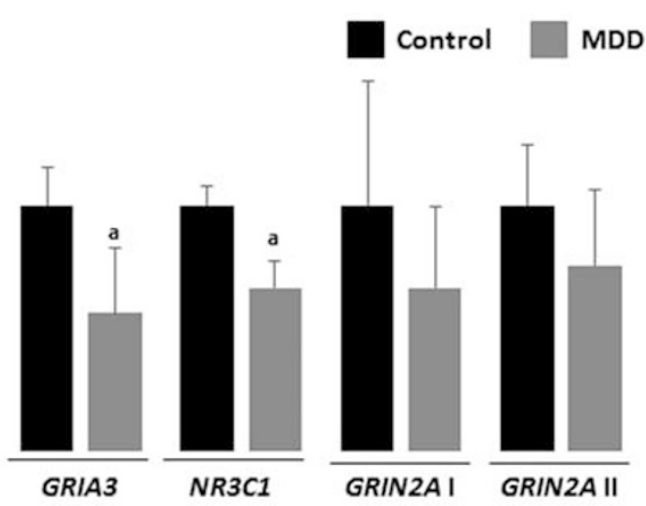

c

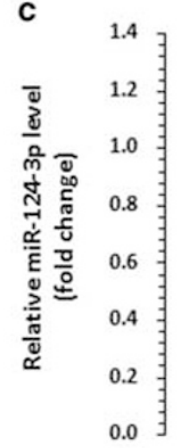

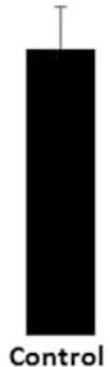

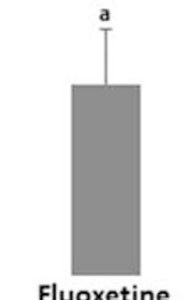

d

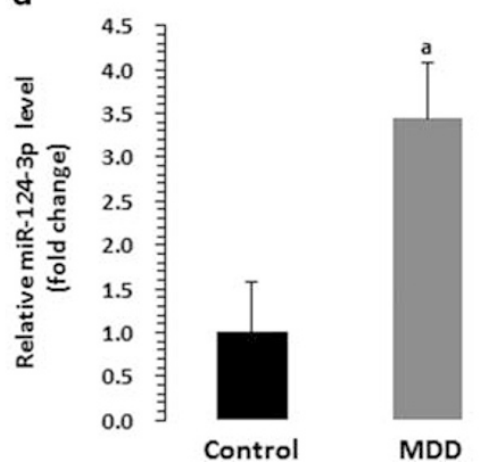

Figure 5 Expression profiling of miR-124-3p and its target genes. (a) Representative graph demonstrates the relative transcript abundance of miR- I24-3p in BA46 area of human post-mortem brain of major depressive disorder (MDD) subjects. Values are expressed as fold increase in expression compared with healthy control ( $n=15$ per group), and data are normalized using U6 gene expression values and represented as \pm SEM. Level of significance was determined using independent-sample ' $t$ '-test. 'a' denotes significant difference between MDD and control groups $(\mathrm{df}=28, f=2.383, t=4.349, p<0.00 \mathrm{I})$. (b) GAPDHnormalized relative expression level of GRIA4, GRIA3, GRIN2A I (representing transcript variants I and 2), GRIN2 II (representing transcript variant 3), and $\mathrm{NR3CI}$ mRNA analyzed in BA46 area of human post-mortem brain tissues suffering from MDD as compared with the normal healthy control group. All data demonstrate the mean \pm SEM (GRIA4 $n=\mid 5$ per group, GRIA3 $n=\mid 5$ per group, NR3CI $n=\mid 5$ control+ $\mid 4$ MDD and GRIN2A I and $\| n=\mid 5$ per group). The level of significance was determined using independent-sample ' $t$ ' test. 'a' denotes significant difference between MDD and control groups (GRIA4 df $=28$, $f=2.204, t=-2.676, p=0.012 ; G R I A 3 d f=28, f=8.7 I I, t=2.685, p=0.012 ; N R 3 C I d f=27, f=0.796, t=2.28 I, p=0.03 ; G R I N 2 A I d f=28, f=1.50 I$, $t=1.026, p=0.31$; and GRIN2A $\| d f=28, f=0.001, t=1.063, p=0.29)$. (c) Graph representing the relative transcript abundance of miR- I24-3p in prefrontal cortex (PFC) of rats treated with fluoxetine. U6-normalized values are expressed as fold change in expression compared with control ( $n=5$ per group), data are represented as \pm SEM, and the level of significance was determined with independent-sample ' $t$ ' test $(p=0.04 I)$. 'a' denotes significant difference between fluoxetine and control groups. (d) Relative transcript abundance of miR- I24-3p normalized with U6 expression represented as fold change in MDD patients $(n=18)$ as compared with healthy controls $(n=17)$. Data represented as \pm SEM and level of significance was determined with independent-sample ' $t$ ' test $(t=2.07, \mathrm{df}=33, p=0.046)$. 'a' denotes significant difference between MDD and control groups.

ionotropic, Ampa4), Grin2a (Glutamate Receptor, Ionotropic, N-Methyl D-Aspartate 2A), Grin2b (Glutamate Receptor, Ionotropic, N-Methyl D-Aspartate 2B), Nr3c1 (Nuclear Receptor Subfamily 3, Group C, Member 1; Glucocorticoid Receptor), Nr3c2 (Nuclear Receptor Subfamily 3, Group C, Member 2; Mineralocorticoid Receptor), Hsp90ab1 (Heat Shock Protein 90kDa Alpha (Cytosolic), Class B Member 1), and $A k t 1 s 1$ (Akt1 Substrate 1). Identification of $N r 3 c 1$ as a direct target of miR-124-3p has been documented in neuronal differentiation process (Vreugdenhil et al, 2009). In the present study, in silico prediction analysis showed that both $N r 3 c 1$ and $N r 3 c 2$ were targets of miR-124-3p with high context scores. Our in vitro cellular model showed downregulation of the $N R 3 C 1$ gene transcript in neuroblastoma cell line (SH-SY5Y) as a function of ectopic overexpression of miR-124-3p and reversal of this attenuation with concomitant knocking down of miR-124-3p. Interestingly, the expression of only $N r 3 c 1$ but not $N r 3 c 2$ gene was decreased in PFC of CORT-treated rats, which was reciprocally correlated with the expression of miR-124-3p expression. This experiment suggests that $N r 3 c 1$ is a bona fide target of miR-124-3p. Further analysis revealed two miR-1243p-binding sites in $3^{\prime}$ UTR of the Nr3c1 gene: 7mer-A1 and 7mer-m8. In vivo 3' UTR enrichment analysis, which identifies direct interaction of miRNAs with putative target genes under physiological conditions, excluded the 7mer-A1binding site, which is proximal to the stop codon. On the other hand, binding site with 7 mer-m8 consensus in $3^{\prime}$ UTR of $N r 3 c 1$, which is distal from stop codon, showed successful binding with miR-124-3p seed sequence. These results suggest that there is an enhanced interaction selectively of 7 mer-m 8 consensus site in $3^{\prime}$ UTR of Nr3cl with miR-124-3p, which could be responsible for the observed repression of the $\mathrm{Nr} 3 \mathrm{cl}$ gene in PFC of CORT-treated rats. The observed miR-124-3pmediated $N r 3 c 1$ repression via the 7 mer-m8 consensusbinding site may be central to behavioral maladaptation and associated neuroendocrine responses to stress and induction of depression phenotype. 
In silico prediction analysis showed that a significant number of AMPA/NMDA receptors are predicted targets of miR-124-3p, suggesting a possible molecular crosstalk between miR-124-3p and these genes. Of these, NMDA receptor subunits Grin $2 a$ and Grin $2 b$ and AMPA receptor subunits Gria3 and Gria4 showed high predictability scores. When examined, we found that expression of Grin $2 a$, Gria3, and Gria4 were significantly reduced in PFC of CORTtreated rats, which established an inverse pattern with the expression of miR-124-3p. On contrary to Grin $2 a$ and Gria3, our in vivo RNP-IP immunoenrichment experiments with Gria4 reflected post-transcriptional repression mediated by miR-124-3p in PFC of CORT-treated rats.

Several studies point to a potential role of glutamatergic system in depression. For example, altered GRIN2A transcript expression has been reported in dlPFC of MDD subjects (Beneyto and Meador-Woodruff, 2008). GRIA3 is well documented for its pre- and post-synaptic localization in the pyramidal neurons of PFC and potential contribution toward synaptic plasticity (Sprengel, 2006). Reports of altered GRIA3 expression in dentate gyrus and CA1 (Duric et al, 2013) as well as in PFC of MDD individuals (MeadorWoodruff et al, 2001; Beneyto and Meador-Woodruff, 2006) raise the possibility of potential contribution of this AMPA receptor in compromised synaptic plasticity in depression. GRIA4, which is presumably involved in synaptic plasticity and the development of functional neural circuitry through the recruitment of other AMPA receptor subunits, has not been well studied in relation to stress or depression, although some studies point to a decreased expression of this receptor in PFC of MDD subjects (Meador-Woodruff et al, 2001; Beneyto and Meador-Woodruff, 2006). More recently, it has been shown that miR-124 and GRIA4 may contribute to social behavioral deficits in frontotemporal dementia (Gascon et al, 2014) and reduced expression of AMPA receptors and gradual loss of post-synaptic membranes of PFC neurons may be critical under chronic stress (Yuen et al, 2012). Although membrane trafficking and ubiquitinationinduced receptor degradation have been shown to be responsible for impaired receptor functionality under chronic stress (Yuen et al, 2012), the precise molecular mechanism is unknown. Our current observations of miR-124-3p-mediated repression of glutamate receptor transcripts open up a new layer of complex regulation of glutamatergic system, which could eventually shed light on underlying epigenetic regulation of glutamatergic neurotransmission as a consequence of hyperactive HPA axis and consequent depression.

Despite strong prediction scores in the 3' UTR of miR-124-3p, perfect 8 mer seed sequence matches, and in vitro expression modulation with miR-124-3p mimic and antisense overexpression, we did not find any significant change in the expression of Hsp90ab1 or Akt1s1 in PFC of CORT-treated rats. Hsp90ab1 is a member of heat shock protein family 90 and has been shown to be activated as a function of glucocorticoid receptor overexpression in mouse hippocampal dentate gyrus (Wei et al, 2012). On the other hand, Akt1s1 is a substrate of Akt1 and is a critical component of mTORC1 signaling (Sancak et al, 2007), whose role in synaptogenesis is well demonstrated (Dwyer and Duman, 2013; Ota et al, 2014). Our findings rule out in vivo interaction of at least the Akt1s1 gene with miR-124 under CORT chronic treatment, suggesting that these genes, although may be important in the stress pathway, their regulation may not be associated directly with miR-124-3p.

To test whether the observed changes in miR-124-3p and target genes can be replicated in MDD patients, we examined their expression levels in PFC obtained from subjects diagnosed with MDD and healthy controls. As with CORT-treated rats, we found that not only the expression of miR-124-3p was upregulated but there was a reciprocal downregulation of target genes GRIA3, GRIA4, and NR3C1 in PFC of MDD subjects. Several reports suggest that under certain pathophysiological conditions, miRNAs are released into extracellular space and appear in the body fluids, which could be due to blebbing of apoptotic bodies, budding, and shedding of microvesicles or due to active secretion in the form of exosomes as complexes with proteins such as AGO2, NPM1, and so on. Because of these reasons, several recent studies show that circulating miRNAs may serve as biomarker for disease pathophysiology (Foss et al, 2011; Zhang et al, 2011; Jin et al, 2013; Sheinerman and Umansky, 2013; Maffioletti et al, 2014; Tsujiura et al, 2015). When investigated, we found that the expression of miR-124-3p was significantly upregulated in the serum of MDD patients compared with healthy control subjects, which was similar to CORT-treated rats and human postmortem studies.

\section{CONCLUSION}

Altogether, this is the first comprehensive and mechanistic study at in vitro and in vivo levels, which demonstrates that not only are there consistent depression-associated changes in the expression of miR-124-3p across different species but also the genes that are targets of this miRNA are highly dysregulated, showing altered response at the functional level. We also identified a specific precursor miRNA (miR-124-3) located on rat chromosome 3, which appears to be responsible for the higher expression of miR-124-3p. Interestingly, miR-124-3p is epigenetically regulated, which for the first time suggests the role played by geneenvironment interaction in miRNA-mediated regulation of gene expression. Our study also has implications in identifying a putative biomarker for MDD pathogenesis as similar changes were noted in the expression of this miRNA in postmortem brain and serum of MDD subjects. This appears to be highly reliable as miR-124-3p can cross the blood-brain barrier and be released into blood (Sheinerman and Umansky, 2013; Devaux et al, 2015). Therapeutically, miR-124-3p can be a potential target for novel drug development, which is supported by a recent observation that downregulating miR-124-3p can lead to resistance in depression-like behavior in rodents (Bahi et al, 2014).

\section{FUNDING AND DISCLOSURE}

The research was supported by grants from the National Institute of Mental Health (R01MH082802; R21MH081099; 1R01MH101890; R01MH100616; and 1R01MH107183), American Foundation for Suicide Prevention (SRG-XXXX001778-1209) to Dr Dwivedi. We sincerely thank the Maryland Brain Collection, Baltimore, USA, for providing human post-mortem brain samples. 


\section{ACKNOWLEDGMENTS}

YD conceived, designed, and coordinated the study. BR performed majority of the in vitro and in vivo studies. MD provided technical support for molecular studies. RCS coordinated patient recruitment, psychiatric diagnosis, and blood samples. YD, BR, MD, and RCS contributed in writing the manuscript.

\section{REFERENCES}

Bahi A, Chandrasekar V, Dreyer JL (2014). Selective lentiviralmediated suppression of microRNA124a in the hippocampus evokes antidepressants-like effects in rats. Psychoneuroendocrinology 46: 78-87.

Barbier E, Tapocik JD, Juergens N, Pitcairn C, Borich A, Schank JR et al (2015). DNA methylation in the medial prefrontal cortex regulates alcohol-induced behavior and plasticity. J Neurosci 35: 6153-6164.

Belmaker RH, Agam G (2008). Major depressive disorder. N Engl J Med 358: 55-68.

Beneyto M, Meador-Woodruff JH (2006). Lamina-specific abnormalities of AMPA receptor trafficking and signaling molecule transcripts in the prefrontal cortex in schizophrenia. Synapse 60: 585-598.

Beneyto M, Meador-Woodruff JH (2008). Lamina-specific abnormalities of NMDA receptor-associated postsynaptic protein transcripts in the prefrontal cortex in schizophrenia and bipolar disorder. Neuropsychopharmacology 33: 2175-2186.

Bromet E, Andrade LH, Hwang I, Sampson NA, Alonso J, de Girolamo G et al (2011). Cross-national epidemiology of DSM-IV major depressive episode. BMC Med 9: 90.

Chandrasekar V, Dreyer JL (2009). microRNAs miR-124, let-7d and miR-181a regulate cocaine-induced plasticity. Mol Cell Neurosci 42: 350-362.

Devaux Y, Stammet P, Friberg H, Hassager C, Kuiper MA, Wise MP et al (2015). MicroRNAs: new biomarkers and therapeutic targets after cardiac arrest? Crit Care 19: 54.

Duric V, Banasr M, Stockmeier CA, Simen AA, Newton SS, Overholser JC et al (2013). Altered expression of synapse and glutamate related genes in post-mortem hippocampus of depressed subjects. Int J Neuropsychopharmacol 16: 69-82.

Dwivedi Y, Rizavi HS, Conley RR, Pandey GN (2006). ERK MAP kinase signaling in post-mortem brain of suicide subjects: differential regulation of upstream Raf kinases Raf-1 and B-Raf. Mol Psychiatry 11: 86-98.

Dwivedi Y, Roy B, Lugli G, Rizavi H, Zhang H, Smalheiser NR (2015). Chronic corticosterone-mediated dysregulation of microRNA network in prefrontal cortex of rats: relevance to depression pathophysiology. Transl Psychiatry 5: e682.

Dwyer JM, Duman RS (2013). Activation of mammalian target of rapamycin and synaptogenesis: role in the actions of rapid-acting antidepressants. Biol Psychiatry 73: 1189-1198.

First M, Spitzer RL, Gibbon M, Williams JBW (2002). Structured Clinical Interview for DSM-IV-TR Axis I Disorders, Research Version, Non-patient edn. Biometric Research. New York State Psychiatric Institute: New York, NY, USA.

Fischbach SJ, Carew TJ (2009). MicroRNAs in memory processing. Neuron 63: 714-716.

Foss KM, Sima C, Ugolini D, Neri M, Allen KE, Weiss GJ (2011). miR-1254 and miR-574-5p: serum-based microRNA biomarkers for early-stage non-small cell lung cancer. J Thorac Oncol 6: 482-488.

Gascon E, Lynch K, Ruan H, Almeida S, Verheyden JM, Seeley WW et al (2014). Alterations in microRNA-124 and AMPA receptors contribute to social behavioral deficits in frontotemporal dementia. Nat Med 20: 1444-1451.
Hall BS, Moda RN, Liston C (2015). Glucocorticoid mechanisms of functional connectivity changes in stress-related neuropsychiatric disorders. Neurobiol Stress 1: 174-183.

Hansen KF, Karelina K, Sakamoto K, Wayman GA, Impey S, Obrietan K (2013). miRNA-132: a dynamic regulator of cognitive capacity. Brain Struct Funct 218: 817-831.

Harrison PJ, Heath PR, Eastwood SL, Burnet PW, McDonald B, Pearson RC (1995). The relative importance of premortem acidosis and postmortem interval for human brain gene expression studies: selective mRNA vulnerability and comparison with their encoded proteins. Neurosci Lett 200: 151-154.

Jankord R, Herman JP (2008). Limbic regulation of hypothalamopituitary-adrenocortical function during acute and chronic stress. Ann N Y Acad Sci 1148: 64-73.

Jin XF, Wu N, Wang L, Li J (2013). Circulating microRNAs: a novel class of potential biomarkers for diagnosing and prognosing central nervous system diseases. Cell Mol Neurobiol 33: 601-613.

Jovicic A, Roshan R, Moisoi N, Pradervand S, Moser R, Pillai B et al (2013). Comprehensive expression analyses of neural cell-typespecific miRNAs identify new determinants of the specification and maintenance of neuronal phenotypes. I Neurosci 33: 5127-5137.

Keene JD, Komisarow JM, Friedersdorf MB (2006). RIP-Chip: the isolation and identification of mRNAs, microRNAs and protein components of ribonucleoprotein complexes from cell extracts. Nat Protoc 1: 302-307.

Kim VN (2005). MicroRNA biogenesis: coordinated cropping and dicing. Nat Rev Mol Cell Biol 6: 376-385.

Labermaier C, Masana M, Muller MB (2013). Biomarkers predicting antidepressant treatment response: how can we advance the field? Dis Markers 35: 23-31.

Lai CY, Lee SY, Scarr E, Yu YH, Lin YT, Liu CM et al (2016). Aberrant expression of microRNAs as biomarker for schizophrenia: from acute state to partial remission, and from peripheral blood to cortical tissue. Transl Psychiatry 6: e717.

LaPlant Q, Vialou V, Covington HE 3rd, Dumitriu D, Feng J, Warren BL et al (2010). Dnmt3a regulates emotional behavior and spine plasticity in the nucleus accumbens. Nat Neurosci 13: 1137-1143.

Li LC, Dahiya R (2002). MethPrimer: designing primers for methylation PCRs. Bioinformatics 18: 1427-1431.

Livak KJ, Schmittgen TD (2001). Analysis of relative gene expression data using real-time quantitative PCR and the 2(-Delta Delta C(T)) Method. Methods 25: 402-408.

Lopez JP, Lim R, Cruceanu C, Crapper L, Fasano C, Labonte B et al (2014). miR-1202 is a primate-specific and brain-enriched microRNA involved in major depression and antidepressant treatment. Nat Med 20: 764-768.

Maffioletti E, Tardito D, Gennarelli M, Bocchio-Chiavetto L (2014). Micro spies from the brain to the periphery: new clues from studies on microRNAs in neuropsychiatric disorders. Front Cell Neurosci 8: 75.

Maiorano NA, Mallamaci A (2010). The pro-differentiating role of miR-124: indicating the road to become a neuron. RNA Biol 7: 528-533.

Makeyev EV, Zhang J, Carrasco MA, Maniatis T (2007). The MicroRNA miR-124 promotes neuronal differentiation by triggering brain-specific alternative pre-mRNA splicing. Mol Cell 27: 435-448.

Malki K, Pain O, Tosto MG, Du Rietz E, Carboni L, Schalkwyk LC (2015). Identification of genes and gene pathways associated with major depressive disorder by integrative brain analysis of rat and human prefrontal cortex transcriptomes. Transl Psychiatry 5: e519.

Meador-Woodruff JH, Hogg AJ Jr., Smith RE (2001). Striatal ionotropic glutamate receptor expression in schizophrenia, bipolar disorder, and major depressive disorder. Brain Res Bull 55: 631-640.

Medina A, Seasholtz AF, Sharma V, Burke S, Bunney W Jr., Myers RM et al (2013). Glucocorticoid and mineralocorticoid 
receptor expression in the human hippocampus in major depressive disorder. J Psychiatr Res 47: 307-314.

Montgomery SA, Asberg M (1979). A new depression scale designed to be sensitive to change. Br J Psychiatry 134: 382-389.

Oka M, Rodic N, Graddy J, Chang LJ, Terada N (2006). CpG sites preferentially methylated by Dnmt3a in vivo. J Biol Chem 281: 9901-9908.

Ota KT, Liu RJ, Voleti B, Maldonado-Aviles JG, Duric V, Iwata M et al (2014). REDD1 is essential for stress-induced synaptic loss and depressive behavior. Nat Med 20: 531-535.

Rajasethupathy P, Fiumara F, Sheridan R, Betel D, Puthanveettil SV, Russo JJ et al (2009). Characterization of small RNAs in Aplysia reveals a role for miR-124 in constraining synaptic plasticity through CREB. Neuron 63: 803-817.

Sagata N, Iwaki A, Aramaki T, Takao K, Kura S, Tsuzuki T et al (2010). Comprehensive behavioural study of GluR4 knockout mice: implication in cognitive function. Genes Brain Behav 9: 899-909.

Salzman S, Endicott J, Clayton P, Winokur G (1983). Diagnostic evaluation after death (DEAD) NIMH. Neurosciences Research Branch: Rockville, MD, USA.

Sancak Y, Thoreen CC, Peterson TR, Lindquist RA, Kang SA, Spooner E et al (2007). PRAS40 is an insulin-regulated inhibitor of the mTORC1 protein kinase. Mol Cell 25: 903-915.

Scott HL, Tamagnini F, Narduzzo KE, Howarth JL, Lee YB, Wong LF et al (2012). MicroRNA-132 regulates recognition memory and synaptic plasticity in the perirhinal cortex. Eur $J$ Neurosci 36: 2941-2948.

Sempere LF, Freemantle S, Pitha-Rowe I, Moss E, Dmitrovsky E, Ambros V (2004). Expression profiling of mammalian microRNAs uncovers a subset of brain-expressed microRNAs with possible roles in murine and human neuronal differentiation. Genome Biol 5: R13.

Sheehan DV, Lecrubier Y, Sheehan KH, Amorim P, Janavs J, Weiller E et al (1998). The Mini-International Neuropsychiatric Interview (M.I.N.I.): the development and validation of a structured diagnostic psychiatric interview for DSM-IV and ICD-10. J Clin Psychiatry 59: 22-33.

Sheinerman KS, Umansky SR (2013). Circulating cell-free microRNA as biomarkers for screening, diagnosis and monitoring of neurodegenerative diseases and other neurologic pathologies. Front Cell Neurosci 7: 150.

Smalheiser NR, Lugli G, Rizavi HS, Torvik VI, Turecki G, Dwivedi Y (2012). MicroRNA expression is down-regulated and reorganized in prefrontal cortex of depressed suicide subjects. PLoS One 7: e33201.

Smalheiser NR, Lugli G, Rizavi HS, Zhang H, Torvik VI, Pandey GN et al (2011). MicroRNA expression in rat brain exposed to repeated inescapable shock: differential alterations in learned helplessness vs. non-learned helplessness. Int J Neuropsychopharmacol 14: 1315-1325.

Smalheiser NR, Zhang H, Dwivedi Y (2014). Enoxacin elevates microRNA levels in rat frontal cortex and prevents learned helplessness. Front Psychiatry 5: 6.

Smirnova L, Grafe A, Seiler A, Schumacher S, Nitsch R, Wulczyn FG (2005). Regulation of miRNA expression during neural cell specification. Eur J Neurosci 21: 1469-1477.

Spitzer RW, Gibbon M, First MD (1995). Structural Clinical Interview for DSM-IV (SCID) Biometrics Research. New York State Psychiatric Institute: New York, NY, USA.

Sprengel R (2006). Role of AMPA receptors in synaptic plasticity. Cell Tissue Res 326: 447-455.

Tsujiura M, Komatsu S, Ichikawa D, Shiozaki A, Konishi H, Takeshita $\mathrm{H}$ et al (2015). Circulating miR-18a in plasma contributes to cancer detection and monitoring in patients with gastric cancer. Gastric Cancer 18: 271-279.

Uhlen M, Fagerberg L, Hallstrom BM, Lindskog C, Oksvold P, Mardinoglu A et al (2015). Proteomics. Tissue-based map of the human proteome. Science 347: 1260419.

Valencia-Sanchez MA, Liu J, Hannon GJ, Parker R (2006). Control of translation and mRNA degradation by miRNAs and siRNAs. Genes Dev 20: 515-524.

Vreugdenhil E, Verissimo CS, Mariman R, Kamphorst JT, Barbosa JS, Zweers T et al (2009). MicroRNA 18 and 124a down-regulate the glucocorticoid receptor: implications for glucocorticoid responsiveness in the brain. Endocrinology 150: $2220-2228$.

Walker RM, Rybka J, Anderson SM, Torrance HS, Boxall R, Sussmann JE et al (2015). Preliminary investigation of miRNA expression in individuals at high familial risk of bipolar disorder. J Psychiatr Res 62: 48-55.

Warden D, Trivedi MH, Wisniewski SR, Davis L, Nierenberg AA, Gaynes BN et al (2007). Predictors of attrition during initial (citalopram) treatment for depression: a $\operatorname{STAR}^{\star} \mathrm{D}$ report. Am J Psychiatry 164: 1189-1197.

Wei Q, Fentress HM, Hoversten MT, Zhang L, Hebda-Bauer EK, Watson SJ et al (2012). Early-life forebrain glucocorticoid receptor overexpression increases anxiety behavior and cocaine sensitization. Biol Psychiatry 71: 224-231.

Yuen EY, Wei J, Liu W, Zhong P, Li X, Yan Z (2012). Repeated stress causes cognitive impairment by suppressing glutamate receptor expression and function in prefrontal cortex. Neuron 73: 962-977.

Zhang HL, Yang LF, Zhu Y, Yao XD, Zhang SL, Dai B et al (2011). Serum miRNA-21: elevated levels in patients with metastatic hormone-refractory prostate cancer and potential predictive factor for the efficacy of docetaxel-based chemotherapy. Prostate 71: 326-331.

Supplementary Information accompanies the paper on the Neuropsychopharmacology website (http://www.nature.com/npp) 